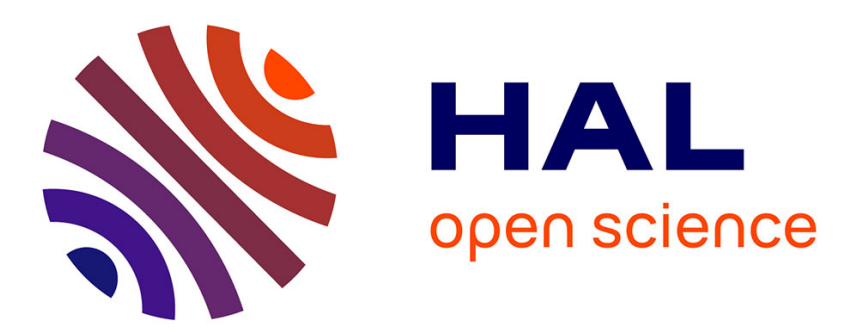

\title{
Photodissociation Electronic Spectra of Cold Protonated Quinoline and Isoquinoline in the Gas Phase
}

Géraldine Feraud, Luis Domenianni, Ernesto Marceca, Claude

Dedonder-Lardeux, Christophe Jouvet

\section{- To cite this version:}

Géraldine Feraud, Luis Domenianni, Ernesto Marceca, Claude Dedonder-Lardeux, Christophe Jouvet. Photodissociation Electronic Spectra of Cold Protonated Quinoline and Isoquinoline in the Gas Phase. Journal of Physical Chemistry A, 2017, 10.1021/acs.jpca.7b01301 . hal-01575636

\section{HAL Id: hal-01575636 \\ https://hal-amu.archives-ouvertes.fr/hal-01575636}

Submitted on 15 May 2018

HAL is a multi-disciplinary open access archive for the deposit and dissemination of scientific research documents, whether they are published or not. The documents may come from teaching and research institutions in France or abroad, or from public or private research centers.
L'archive ouverte pluridisciplinaire $\mathbf{H A L}$, est destinée au dépôt et à la diffusion de documents scientifiques de niveau recherche, publiés ou non, émanant des établissements d'enseignement et de recherche français ou étrangers, des laboratoires publics ou privés. 


\title{
Photodissociation Electronic Spectra of Cold Protonated Quinoline and Isoquinoline in the Gas-Phase
}

\author{
Géraldine Féraud $^{\mathrm{a}, \mathrm{c}}$; Luis Domenianni ${ }^{\mathrm{b} 1, \mathrm{~b} 2}$; Ernesto Marceca ${ }^{\mathrm{b} 1, \mathrm{b2} 2^{*}}$; Claude Dedonder- \\ Lardeux $^{\mathrm{a}}$; Christophe Jouvet ${ }^{\mathrm{a}^{*}}$
}

\footnotetext{
a) CNRS, Aix-Marseille Université, PIIM UMR 7365, Avenue Escadrille Normandie-Niémen, 13397 Marseille Cedex 20, France

${ }^{\text {b1) }}$ Universidad de Buenos Aires, Facultad de Ciencias Exactas y Naturales, DQIAF, Ciudad Universitaria, $1^{\text {er }}$ piso, Pab. II, C1428EGA, Buenos Aires, Argentina

${ }^{\text {b2) }}$ CONICET-UBA, Inst. Química Física de los Materiales, Medio Ambiente y Energía (INQUIMAE), Ciudad Universitaria, $3^{\text {er }}$ piso, Pab. II, C1428EGA, Buenos Aires, Argentina

c) Present address: UPMC, Pierre and Marie Curie University, Paris 6, 4 Place Jussieu, 75005 Paris, France

* corresponding authors: christophe.jouvet@univ-amu.fr, marceca@qi.fcen.uba.ar
}

\begin{abstract}
:
Photofragmentation electronic spectra of isolated single-isomeric N-protonated quinoline (quinolinium) and isoquinoline (isoquinolinium) ions have been measured at a temperature of $\sim 40 \mathrm{~K}$ using a mass-selective, $10 \mathrm{~cm}^{-1}$ spectral resolution, photodissociation spectrometer. Additionally, ab-initio adiabatic transition energies calculated using the RI-ADC(2) method have been employed to assist in the assignment of the spectra. Three electronic transitions having $\pi \pi^{*}$ character were clearly evidenced for both protonated ions within the UV and deep-UV spectral ranges. The corresponding spectra at room temperature were previously reported by Hansen et al., ${ }^{24}$ together with TD-DFT calculations and a careful analysis of the possible fragmentation mechanisms. This information will be complemented in the present study by appending better resolved spectra, characteristic of cold ions, in which well-defined vibrational progressions associated to the $\mathrm{S}_{1} \leftarrow \mathrm{S}_{0}$ and $\mathrm{S}_{3} \leftarrow \mathrm{S}_{0}$ transitions exhibit clear 0-0 bands at $\mathrm{h} v_{0-0}=27868$ and $42230 \mathrm{~cm}^{-1}$, for protonated quinoline, and at $\mathrm{h} v_{0-0}=28043$ and $41988 \mathrm{~cm}^{-1}$, for protonated isoquinoline. Active vibrations in the spectra were assigned with the help of calculated normal modes, looking very similar to those of the structurally related protonated naphthalene. Finally, we have observed that the bandwidths associated with the deep-UV $S_{3} \leftarrow S_{0}$ transition denote a lifetime for the $S_{3}$ excited state in the subpicosecond time scale, in contrast with that of $\mathrm{S}_{1}$.
\end{abstract}




\section{Introduction:}

Polycyclic aromatic nitrogen heterocycles (PANHs) involve a group of compounds widely distributed in the Earth's biosphere ${ }^{1}$ as a consequence of the emission caused by natural fires, incomplete combustion of fuels and discharge of industrial effluents, among other sources. ${ }^{2,3}$ The toxicity of several PANHs, ${ }^{4}$ normally exceeding that of the structurally related polycyclic aromatic hydrocarbons (PAHs), has motivated the development of analytical methods that, in many cases, must rely on the scarce photophysical information existing for these molecules. ${ }^{5}$

Additionally, many authors have hypothesized ${ }^{6-8}$ that PANHs might also exist beyond the Earth throughout the interstellar medium (ISM), as a result of the reaction between interstellar nitrogen and PAHs. The presence of PAHs in the ISM has long been postulated to account for absorption features in the diffuse interstellar bands (DIBs) ${ }^{9-11}$ and emission features in the aromatic infrared bands (AIBs). ${ }^{12}$ More recently, it was pointed out using experimental and theoretical arguments that the AIBs' assignment is improved by considering nitrogen-containing PAHs. ${ }^{8,13-16}$ Nevertheless, very few gas phase electronic spectra are available to conclusively establish whether the visible absorption of $\mathrm{PANH}^{+} \mathrm{s}$ is part of the observed astronomical DIBs. ${ }^{17}$ In addition to the radical cations, protonated PANHs (hereafter $\mathrm{PANHH}^{+} \mathrm{s}$ ) will likely be found in the ionized environments of the ISM due to the high proton affinity of the aromatic nitrogen expected for these molecules. Evidence supporting this assumption was given by recent IR measurements ${ }^{15}$ performed on four small-size $\mathrm{PANHH}^{+} \mathrm{s}$ that were found to reproduce closely the $6.2 \mu \mathrm{m}$ and $8.6 \mu \mathrm{m}$ AIBs. However, well resolved electronic spectra of isolated $\mathrm{PANHH}^{+} \mathrm{s}$ are still needed ${ }^{18}$ to explore if the visible absorption of these ions is a component of the DIBs.

In this work we have measured the photodissociation electronic spectra of cold protonated quinoline and isoquinoline, the two simplest $\mathrm{PANHH}^{+} \mathrm{s}$ comprising a pyridinium cation fused to a six-membered carbocyclic ring. Both quinoline and isoquinoline are structurally related to naphthalene, being one $\mathrm{CH}$ group of the latter molecule substituted by one aromatic nitrogen atom at positions 1 and 2, respectively (Scheme 1). Protonation occurs on the nitrogen $\mathrm{sp}^{2}$ lone electron pair leading to the corresponding quinolinium $\left(\mathrm{QH}^{+}\right)$or isoquinolinium $\left(\mathrm{iQH}^{+}\right)$cations, as shown in Scheme 1. C-protonation is highly disfavored due to the electron deficiency at the aromatic carbons caused by the inductive polarization of the electronegative heteroatom. 

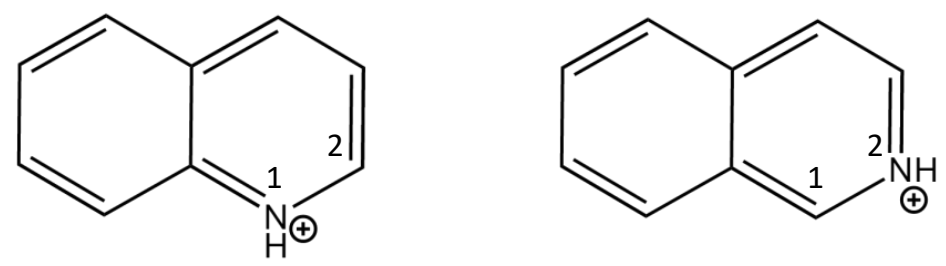

Scheme 1. Left, protonated quinoline (quinolinium ion, $\mathrm{QH}^{+}$); right, protonated isoquinoline (isoquinolinium ion, $\mathrm{iQH}^{+}$).

Even though the electronic spectroscopic properties of neutral quinolines ${ }^{19-22}$ and their radical cations ${ }^{17}$ have been extensively studied in the gas phase, less information is available on the corresponding isolated protonated molecules. ${ }^{23,24}$ In particular, only one spectrum at room temperature has been obtained for $\mathrm{QH}^{+}$and $\mathrm{iQH}^{+}$using photodissociation action spectroscopy by Hansen et al., ${ }^{24}$ and so far well resolved vibronic spectra of cold ions are still lacking. A careful analysis of the fragmentation mechanism has been done in the paper by Hansen et al., and we will use their calculations for further discussion. We will present new $\mathrm{UV}$-vis spectroscopic data for $\mathrm{QH}^{+}$and $\mathrm{iQH}^{+}$in which we were able to improve the accuracy of the electronic band origins, exhibit well resolved vibrational progressions associated to the low lying excitations, and provide estimations about the lifetime of the excited states based on the observed line broadenings. All the measurements were performed using a cryogenic ion trap photodissociation spectrometer in which the ions can be cooled to temperatures of a few tens of Kelvin. Pump-probe UV-UV ion-dip experiments ${ }^{25}$ were employed to explore the contribution of possible tautomers in the spectral bands.

The photochemical processes observed for protonated quinoline and isoquinoline will be compared with former measurements on protonated naphthalene. ${ }^{26,27}$ To support the spectroscopic observations, ab-initio calculations were performed on $\mathrm{QH}^{+}$and $\mathrm{iQH}^{+}$ground and excited states.

\section{Methods:}

Electronic spectra were recorded using the same experimental set-up as in previous work. $^{28,29}$ Only a brief description of the apparatus will be given here, including the ions' preparation method and the applied spectroscopic techniques. Parent $\mathrm{QH}^{+}$and $\mathrm{iQH}^{+}$ions are formed in an electrospray source fed by a continuum flow of an acidulated water/methanol mixture (1:1 in volume) in which the corresponding quinoline is dissolved at a concentration 
of $0.1 \mathrm{mM}$. A mass gate controls the injection of positively charged particles into a cryogenically-cooled Paul trap, where they are stored for several tens of milliseconds. During this lapse of time the internal temperature of the ions is drastically reduced to about $40 \mathrm{~K}$ by collisions with cold He atoms, previously pulsed into the trap. Next, a fraction of the cold parent ions is photodissociated by a first laser pulse mildly focused in the center of the trap to a spot of $\sim 2 \mathrm{~mm}^{2}$, and all the ions (ionic fragments and the surviving parent ions) are extracted into a linear time of flight (TOF) mass spectrometer where they are mass-analyzed. Photofragmentation electronic spectra are obtained by plotting the ion signal corresponding to each mass channel vs. the wavelength of the fragmentation laser. In auxiliary experiments, the ion extraction can be gradually delayed in order to monitor the time evolution of the amount of fragments in the trap, with the purpose of studying if different fragmentation paths are active. Laser wavelengths are selected within $10 \mathrm{~cm}^{-1}$ by use of two optical parametric oscillators (EKSPLA, model-NT342B) that operate with scanning steps up to $0.02 \mathrm{~nm}$, pulse energies of $\sim 0.5 \mathrm{~mJ}$ and a duty cycle of $10 \mathrm{~Hz}$.

Additionally, a double resonance method ${ }^{29}$ can be applied to a determined ion in the trap in order to discriminate whether the observed spectral bands belong to a unique species (different transitions sharing the same ground state) or originate from different conformers (further details in the Supporting Information file).

Ab-initio calculations were performed using the TURBOMOLE package, ${ }^{30}$ following the resolution-of-the-identity (RI) approximation to calculate the electron repulsion integrals. The ground state $\left(\mathrm{S}_{0}\right)$ geometries of $\mathrm{QH}^{+}$and $\mathrm{iQH}^{+}$were optimized at the MP2/cc-pVDZ level (second order Møller-Plesset perturbation theory, with the correlation-consistent polarized valence double-zeta basis set). Vertical transitions energies and oscillator strengths of the lowest excited singlet states $\left(\mathrm{S}_{1-3}\right)$ were calculated using the RI-ADC(2) (second order Algebraic Diagrammatic Construction) method, ${ }^{31}$ which was previously found to reproduce adequately the electronic excitations of other ionic systems containing aromatic nitrogen atoms, ${ }^{18,32,33}$ as well as protonated aromatic molecules having nitrogen functional groups. ${ }^{34}$

Computations were performed in $\mathrm{C}_{1}$ and $\mathrm{Cs}$ point-group symmetries. Under Cssymmetry restriction, the $\mathrm{S}_{1}$ excited states of $\mathrm{QH}^{+}$and $\mathrm{iQH}^{+}$are found of the $\mathrm{A}^{\prime}\left(\pi \pi^{*}\right)$ type. Supplementary excited state optimizations starting from non-planar structures returned back to the planar geometry. Ground and excited state zero-point energies (ZPEs) have been calculated for the two protonated quinolines to correct the computed transition energy values. 
The vibrational frequencies associated to the ground and the excited states of the $\mathrm{QH}^{+}$and $\mathrm{iQH}^{+}$ions have been calculated to help us assign the experimental vibronic bands. Mulliken notation was used to label the calculated normal modes.

\section{Results:}

\subsection{Electronic spectra of $\mathrm{QH}^{+}$}

In Figure 1 it is presented the difference TOF mass spectra obtained by subtraction of the ion signal corresponding to post- and pre-photodissociation of the parent ion $\mathrm{QH}^{+}(\mathrm{m} / \mathrm{q}=$ 130 , negative peak) at fragmentation energies corresponding to $\mathrm{h} v=42230 \mathrm{~cm}^{-1}(236.8 \mathrm{~nm})$ and $\mathrm{h} v=27871 \mathrm{~cm}^{-1}(358.8 \mathrm{~nm})$. According to the observation of Hansen et al., ${ }^{24}$ the most significant signals (positive peaks) found in the photofragmentation mass spectra for an excitation energy between 41600-43500 $\mathrm{cm}^{-1}(230-240 \mathrm{~nm})$, at room temperature, correspond to $m / q=77$ ions (phenyl cations), formed by either loss of acetylene and hydrogen cyanide molecules or loss of $\mathrm{C}_{3} \mathrm{H}_{3} \mathrm{~N}$ (acrylonitrile or azete), and a smaller yield of $\mathrm{m} / q=102$ ions, resulting from elimination of 28 mass units given by loss of acetylene $+\mathrm{H}_{2}$ or $\mathrm{CN}+\mathrm{H}_{2}$. If the fragmentation energy is decreased to wavelengths in the spectral region of 27800-29400 $\mathrm{cm}^{-1}(340-360 \mathrm{~nm})$, and hence the dissociation process evolves in a different excited state, a drastic change in the branching ratio is observed in the mass spectrum, again in agreement with reference 24 . Under such conditions the most abundant fragment observed is the $\mathrm{m} / \mathrm{q}=$ 103 ion, generated from loss of a hydrogen cyanide molecule, together with two additional minor fragments: $m / q=128$ (loss of $\mathrm{H}_{2}$ or two hydrogen atoms) and $m / q=77$ (phenyl ions). A similar change in the fragmentation pattern is found when $\mathrm{QH}^{+}$is studied by collision induced dissociation (CID) mass spectrometry on going from collision energies of about 50 $\mathrm{eV}$ (main fragment, $m / q=77$ ) to $30 \mathrm{eV}$ (main fragment, $m / q=103$ ). ${ }^{35}$ 


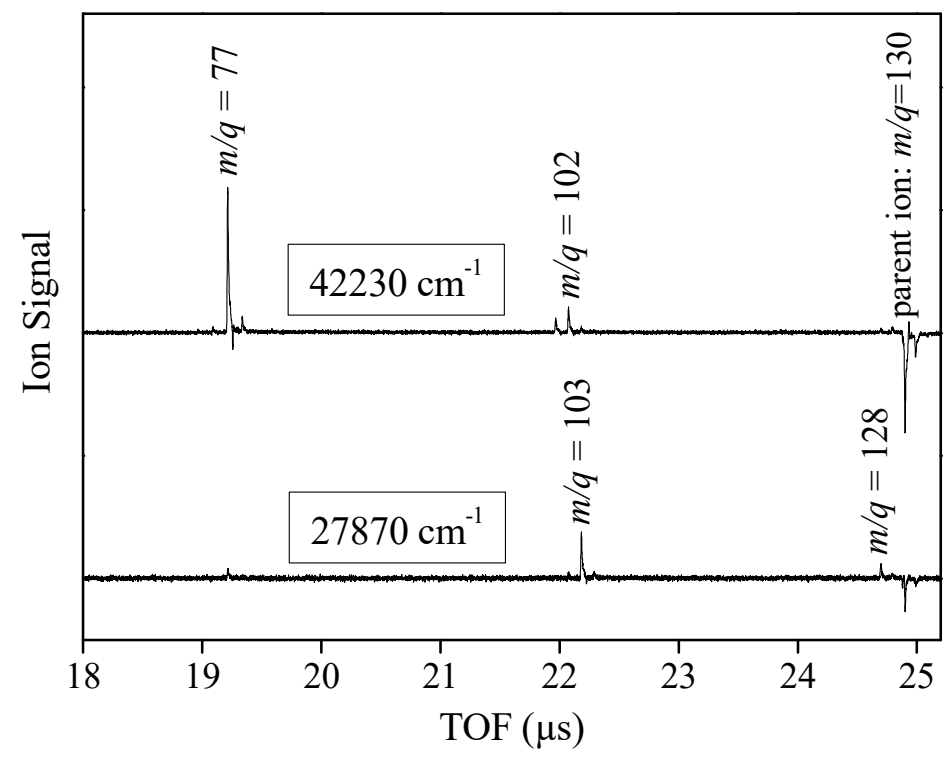

Figure 1. Difference (photodissociation laser: on - off) TOF mass spectra of protonated quinoline $(m / q=130$, negative peak $)$ at $\mathrm{h} v=42230$ and $27870 \mathrm{~cm}^{-1}$. Ion fragment signals (positive peaks) at $m / q=77$ (phenyl cation), 102, 103 and 128 result respectively from loss of $\mathrm{HCN}+\mathrm{C}_{2} \mathrm{H}_{2}$ (or $\mathrm{C}_{3} \mathrm{H}_{3} \mathrm{~N}$ ), $\mathrm{C}_{2} \mathrm{H}_{2}+\mathrm{H}_{2}$ (or $\left.\mathrm{CN}+\mathrm{H}_{2}\right), \mathrm{HCN}$ and $\mathrm{H}_{2}$.

The photodissociation electronic spectra of cold $\mathrm{QH}^{+}$measured by collecting the fragment ion signal on mass channels $m / q=77,102,103$ and 128 are plotted in Figure 2. The onset of the absorption is more clearly visualized on the $m / q=103$ and 128 channels associated to the fragment ions that dominate the mass spectrum upon excitation at lower energy. Three band systems are identified in the spectra. The first band evidences a clear $0-0$ transition at $\mathrm{h} v=27868 \mathrm{~cm}^{-1}$ followed by a well-defined vibrational structure, where the bandwidths are found only slightly broader $\left(15 \mathrm{~cm}^{-1}\right)$ than the spectral resolution of the laser, which should correspond to the convolution of the laser width with the rotational contour. A second absorption appears quite unresolved starting at $\sim 32500 \mathrm{~cm}^{-1}$, which is more clearly distinguished on the $m / q=77$ and 102 channels. From the onset of this band it is possible to estimate a transition energy in the range $32000-33000 \mathrm{~cm}^{-1}$, which agrees perfectly well with the second excited state $\left(\mathrm{S}_{2}\right)$ energy calculated for $\mathrm{QH}^{+}$(see Table 1). The third transition comes into view at $\mathrm{h} v=42230 \mathrm{~cm}^{-1}$, preferentially leading to phenyl fragments $(\mathrm{m} / \mathrm{q}=77)$. It exhibits a rather intense $0-0$ transition together with several active vibrational modes. In this 
third electronic state, however, the bandwidths are markedly larger than those associated with the first excited state (FWHMs $\approx 30 \mathrm{~cm}^{-1}$ ). An interesting feature in the photodissociation spectrum of $\mathrm{QH}^{+}$is the closing of the fragmentation channel corresponding to the elimination of hydrogen cyanide above $\mathrm{h} v=33600 \mathrm{~cm}^{-1}$, as observed in the spectrum recorded in the $\mathrm{m} / \mathrm{q}$ $=103$ fragment (Figure 2).

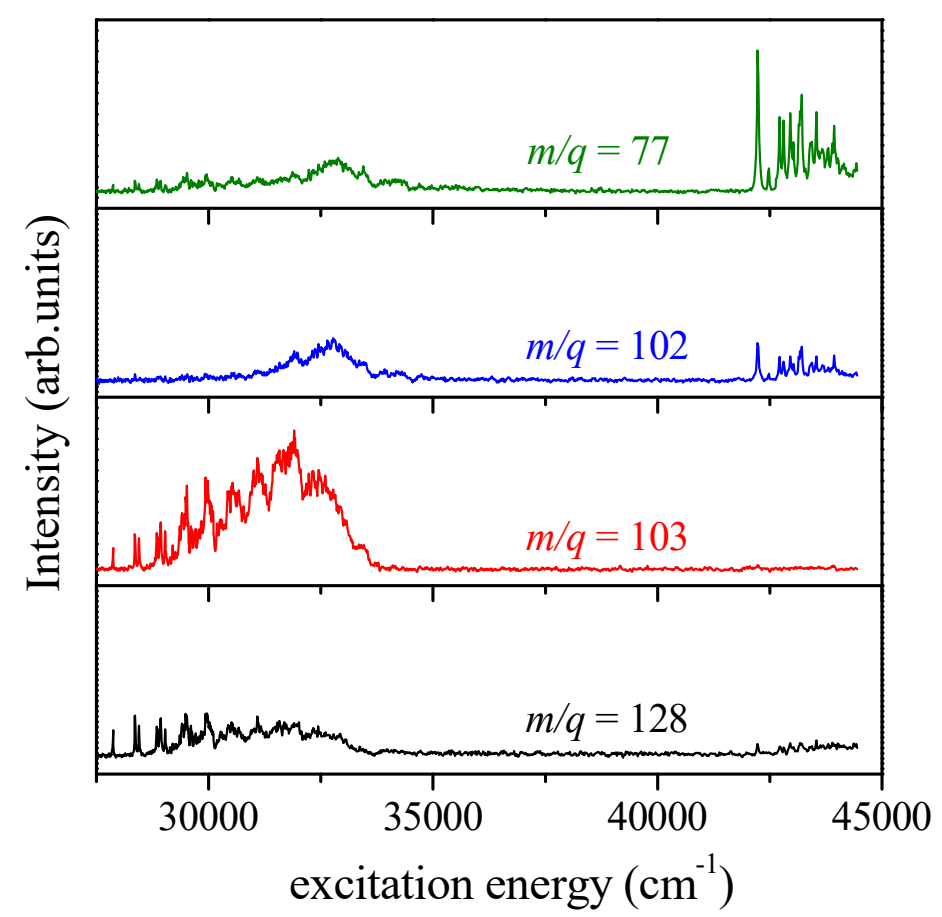

Figure 2. Photofragmentation electronic spectra of protonated quinoline measured by collecting the ion signal on mass channels $m / q=77,102,103$ and 128. Three band systems are observed: i) structured band system starting at $\mathrm{h} v=27868 \mathrm{~cm}^{-1}$ (with decreasing intensities in mass channels $m / q=103$, 128 and 77), ii) unresolved band starting at $\sim 32500 \mathrm{~cm}^{-1}$ (better distinguished on the $m / q=77$ and 102 channels), and iii) structured band system starting at $\mathrm{h} v=42230 \mathrm{~cm}^{-1}(\mathrm{~m} / q=77$ and 102 channels $)$.

The assignment of the vibrational structure of the first and third absorption bands is shown in Figure 3 by zooming respectively the spectral region in the vicinity of $S_{1} \leftarrow S_{0}$ and $\mathrm{S}_{3} \leftarrow \mathrm{S}_{0}$ transition origins. The active vibrations were labeled using the index of the calculated normal mode that better matches the experimental energy (see Table 2). The $\mathrm{S}_{1} \leftarrow \mathrm{S}_{0}$ spectrum of $\mathrm{QH}^{+}$is vibrationally well resolved, having a more complex structure than expected from 
the photodissociation action spectrum measured at room temperature. ${ }^{25}$ It is dominated by three active modes observed at $481 \mathrm{~cm}^{-1}$ (1 and 2 quanta, assigned to $\left.\tilde{v}_{32}\right), 492 \mathrm{~cm}^{-1}$ (1 to 4 quanta, assigned to $\tilde{v}_{31}$ ) and $576 \mathrm{~cm}^{-1}$ (1 to 3 quanta, assigned to $\tilde{v}_{30}$ ), as well as several of their combination bands. The modes $\tilde{v}_{33}, \tilde{v}_{29}$ and $\tilde{v}_{28}$ are also visible in the spectrum and are excited in combination with some of the other active modes. Even though the $\mathrm{S}_{3} \leftarrow \mathrm{S}_{0}$ vibrations are notably broader, we were able to assign vibrational progressions of modes observed at $\tilde{v}_{31}=480 \mathrm{~cm}^{-1}$ (1-2 quanta), $\tilde{v}_{30}=578 \mathrm{~cm}^{-1}$ (1-3 quanta) and $\tilde{v}_{29}=719 \mathrm{~cm}^{-1}(1-2$ quanta), as well as combination bands involving these modes.

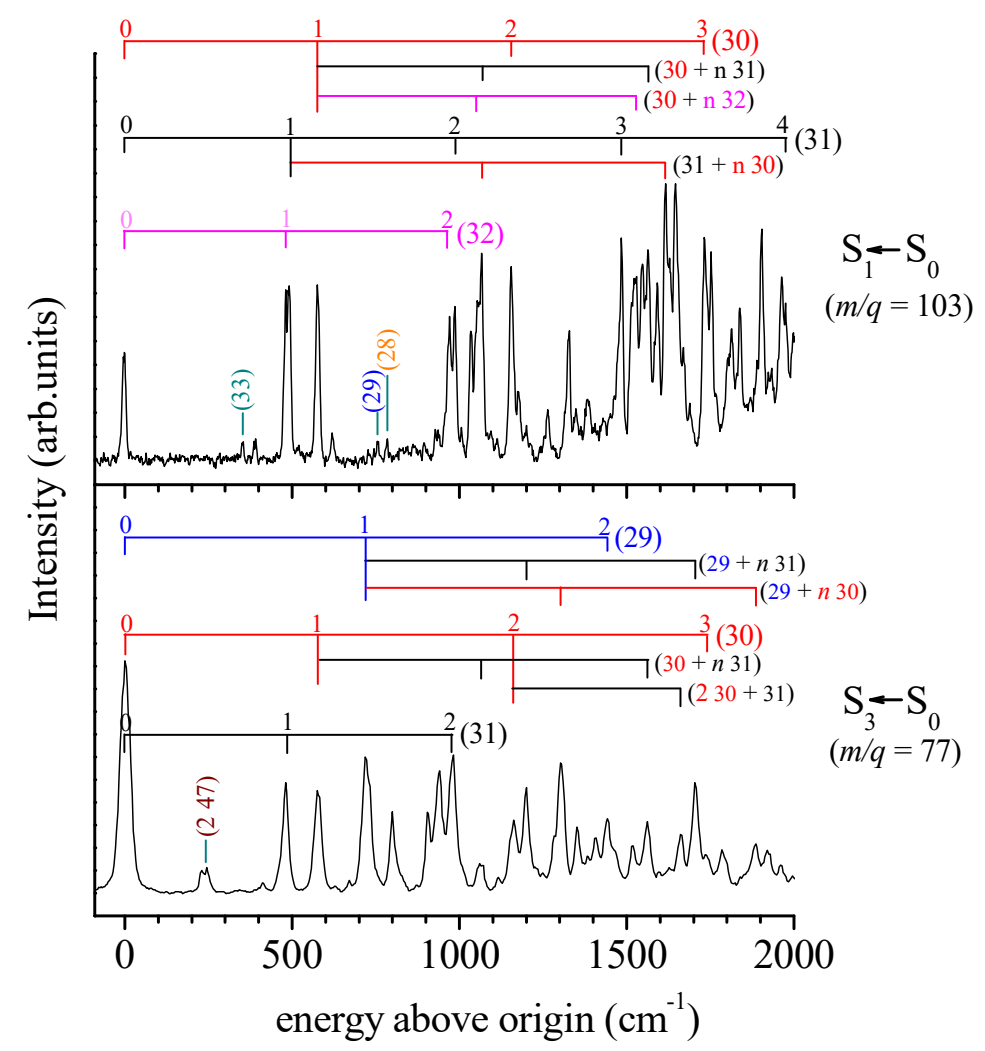

Figure 3. Photofragmentation spectra of cold protonated quinoline in the vicinity of the first $\left(\mathrm{S}_{1} \leftarrow \mathrm{S}_{\mathrm{o}}\right)$ and third $\left(\mathrm{S}_{3} \leftarrow \mathrm{S}_{\mathrm{o}}\right)$ band origins. The spectra were recorded with a $0.02 \mathrm{~nm}$ scanning step on the indicated mass channels. Vibronic assignment of the active modes in the spectra was done with the help of $S_{1}$ and $S_{3}$ calculated normal modes (see Table 2). 


\subsection{Electronic spectra of $\mathrm{iQH}{ }^{+}$}

In agreement with the experiment performed by Hansen et al., ${ }^{24}$ photodissociation mass spectra of trapped $\mathrm{QH}^{+}$and $\mathrm{iQH}^{+}$protonated ions are very much alike, leading to the same fragmentation pattern. For protonated isoquinoline, the main fragment observed via excitation in the $41670-43480 \mathrm{~cm}^{-1}$ energy region is again the phenyl cation $(\mathrm{m} / \mathrm{q}=77)$, changing the branching ratio in favor of the hydrogen cyanide loss upon excitation in the $27780-29410 \mathrm{~cm}^{-1}$ region with the consequent predominance of $m / q=103$ fragments in the mass spectrum. The same behavior is found in CID mass spectra of $\mathrm{iQH}^{+}$for collision energies increasing from 30 to $50 \mathrm{eV}$, where the most intense fragment peak changes from $m / q=103$ to $m / q=77.35$

The UV-vis absorption of cold $\mathrm{iQH}^{+}$is given in Figure 4 for the mass channels $m / q=$ 77, 102, 103 and 128. At first sight, the electronic spectrum looks very similar to that of $\mathrm{QH}^{+}$ and the band origins almost coincide. At low energies, the band recorded on the $m / q=103$ mass channel exhibits a strong $0-0$ transition at $\mathrm{h} v=28043 \mathrm{~cm}^{-1}(356.6 \mathrm{~nm})$ while at higher energies a new band starts at $\mathrm{h} v=41988 \mathrm{~cm}^{-1}(238.2 \mathrm{~nm})$ on the $m / q=77$ channel. Similarly to the $\mathrm{QH}^{+}$case, an intermediate unresolved band appears in the $m / q=77$ channel, this time at $\sim 35100 \mathrm{~cm}^{-1}$, which compares well with the second excited state $\left(\mathrm{S}_{2}\right)$ energy calculated for $\mathrm{iQH}^{+}$(see Table 1) and is shifted by $\sim 2500 \mathrm{~cm}^{-1}$ with respect to the $\mathrm{QH}^{+}$second excited state. Just as in the case of $\mathrm{QH}^{+}$, the fragmentation channel leading to the elimination of hydrogen cyanide $(m / q=103$ mass channel in Figure 4$)$ closes above $~ 33600 \mathrm{~cm}^{-1}$ for iQH . 


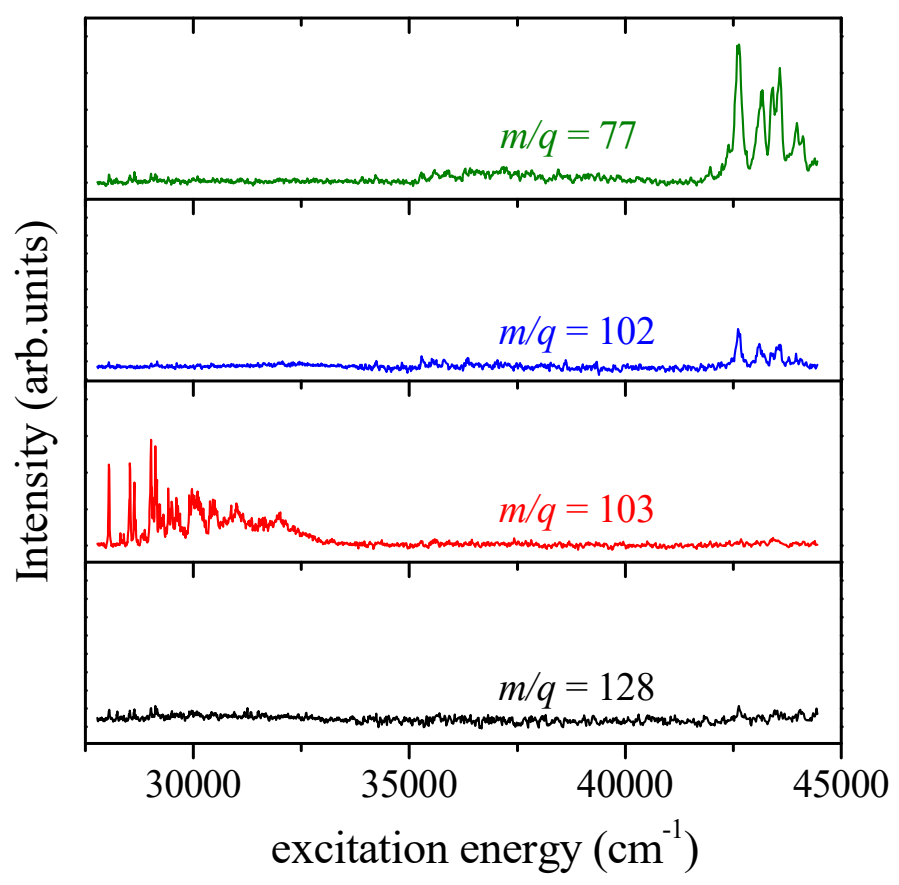

Figure 4. Photofragmentation electronic spectra of protonated isoquinoline measured by collecting the ion signal on mass channels $m / q=77,102,103$ and 128. Three band systems are observed: i) structured band system at $\mathrm{h} v=28043$ $\mathrm{cm}^{-1}$ (clearly observed on the $m / q=103$ mass channel and more weakly for $m / q=77$ ), ii) unresolved band starting at $\sim 35100 \mathrm{~cm}^{-1}$ (better distinguished on the $m / q=77$ and 102 channels), and iii) structured band system at $\mathrm{h} v=41988$ $\mathrm{cm}^{-1}(\mathrm{~m} / \mathrm{q}=77$ and 102 channels $)$.

The first absorption band $\left(\mathrm{S}_{1} \leftarrow \mathrm{S}_{0}\right.$ transition) of $\mathrm{iQH}^{+}$shown in Figure 5 exhibits a well-defined vibrational structure with bandwidths of FWHMs $\approx 15 \mathrm{~cm}^{-1}$, again slightly broader than the spectral resolution of the laser, which should correspond to the convolution of the laser width with the rotational contour. It is possible to distinguish two dominant modes at $\tilde{v}_{31}=492 \mathrm{~cm}^{-1}$ (1-3 quanta), and $\tilde{v}_{30}=589 \mathrm{~cm}^{-1}$ (1-2 quanta), and combination bands between them and including the modes $\tilde{v}_{33}$ and $\tilde{v}_{32}$ as well. Bands corresponding to the modes $\tilde{v}_{33}, \tilde{v}_{32}, \tilde{v}_{29}$ and $\tilde{v}_{28}$ have also been assigned in the spectrum. In the same figure it is observed that the vibrational bands linked to the $\mathrm{S}_{3} \leftarrow \mathrm{S}_{0}$ electronic transition are markedly broader (FWHMs $\approx 130 \mathrm{~cm}^{-1}$ ), which implies that the lifetime of this excited state $\left(\mathrm{S}_{3}\right.$ in our calculations) is limited to less than $\sim 260 \mathrm{fs}$, in contrast to the $\mathrm{S}_{1}$ case. In spite of the large 
bandwidths, we have tentatively assigned two vibrational modes (1-3 quanta) at $\tilde{v}_{32}=461$ $\mathrm{cm}^{-1}$ and $\tilde{v}_{30}=599 \mathrm{~cm}^{-1}$.

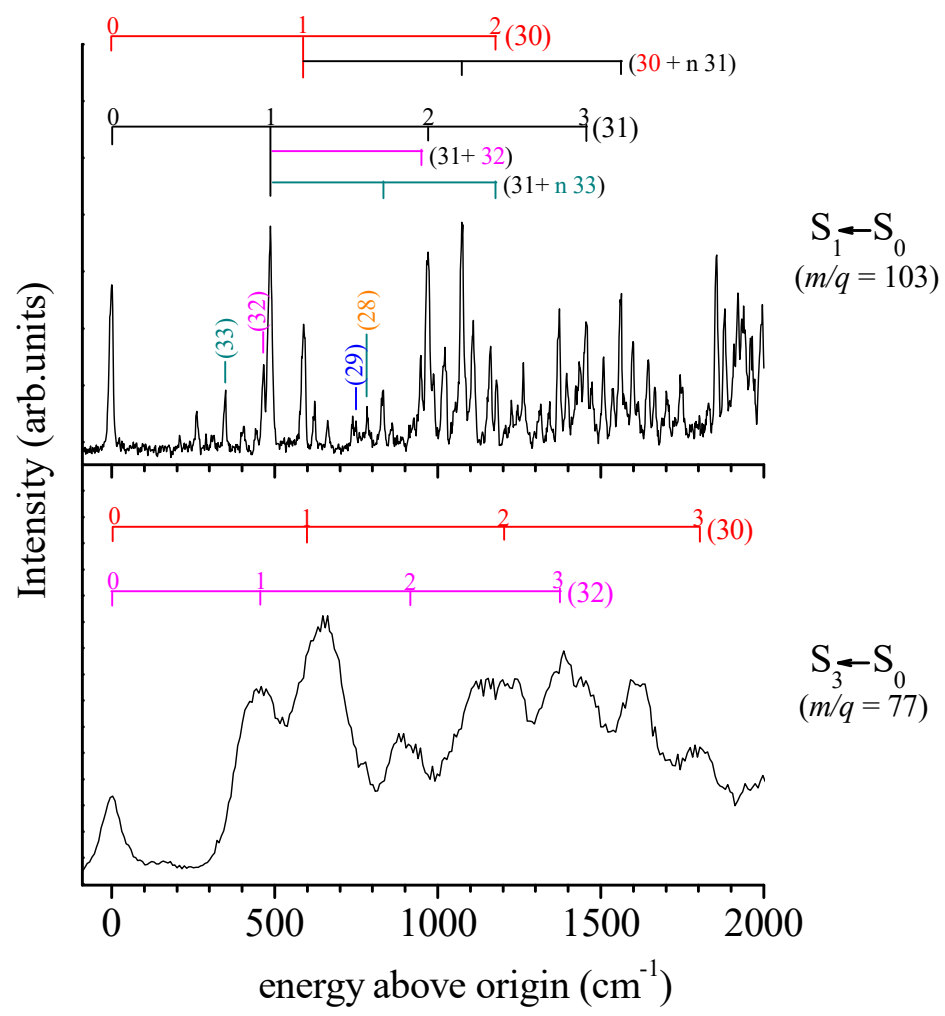

Figure 5. Photofragmentation spectra of cold protonated isoquinoline in the vicinity of the first $\left(\mathrm{S}_{1} \leftarrow \mathrm{S}_{\mathrm{o}}\right)$ and third $\left(\mathrm{S}_{3} \leftarrow \mathrm{S}_{\mathrm{o}}\right)$ band origins. The spectra were recorded with a $0.02 \mathrm{~nm}$ scanning step on the indicated mass channels. Vibronic assignment of the active modes in the spectra was done with the help of $S_{1}$ and $S_{3}$ calculated normal modes (see Table 3 ).

\subsection{Calculations}

The protonation site of $\mathrm{QH}^{+}$and $\mathrm{iQH}^{+}$is limited in our calculations to the $\mathrm{N}$ heteroatom (see Scheme 1). The other tautomers with protonation on a carbon atom are at least $12100 \mathrm{~cm}^{-1}(1.5 \mathrm{eV})$ higher in energy according to our calculations. It is worth to remark that high-energy tautomers cannot usually be ignored since they have been occasionally evidenced $^{28,33}$ despite the low temperatures achieved in the trap. In the present case, Cprotonated tautomers were not taken into account based on the results of double resonance experiments (see Supporting Information), which show that only a single species (Nprotonated tautomer) is responsible for the observed spectra. 
The optimized (MP2/cc-pVDZ) ground state of protonated isoquinoline is $72 \mathrm{~cm}^{-1}$ $(0.009 \mathrm{eV})$ more stable than that of protonated quinoline. $\mathrm{C}_{1}$ point-group symmetry $\mathrm{ADC}(2)$ adiabatic transition energies corrected by the variation of the ZPEs between the excited and the ground states, $\left(E_{\mathrm{o}}\right)$, and the respective oscillator strength values $(f)$ are presented in Table 1 for both ions. Important differences are found between vertical (not shown in Table 1) and adiabatic transition energies, reaching 3600 and $2600 \mathrm{~cm}^{-1}(0.45 \mathrm{eV}$ and $0.32 \mathrm{eV})$ for the corresponding $\mathrm{S}_{1} \leftarrow \mathrm{S}_{0}$ transition in $\mathrm{QH}^{+}$and $\mathrm{iQH}^{+}$, respectively. It points out the necessity of performing excited-state optimizations for these molecular ions in order to properly compare the experimental results with the calculations. It is observed in Table 1 that the ADC(2) energy values computed in the present work are in better correspondence with the experimental band onsets $\left(E_{\exp }\right)$ than the DFT calculations performed previously, ${ }^{24}$ included in the table between brackets.

\begin{tabular}{|c|c|c|c|c|c|c|}
\cline { 2 - 7 } \multicolumn{1}{c|}{} & \multicolumn{3}{c|}{$\mathbf{Q H}^{+}$} & \multicolumn{3}{c|}{$\mathbf{Q H}^{+}$} \\
\cline { 2 - 7 } \multicolumn{1}{c|}{} & $\boldsymbol{E}_{\mathbf{0}} / \mathbf{c m}^{\mathbf{- 1}}$ & $\boldsymbol{E}_{\text {exp } /} \mathbf{c m}^{\mathbf{- 1}}$ & $\boldsymbol{f}$ & $\boldsymbol{E}_{\mathbf{0}} / \mathbf{c m}^{-\mathbf{1}}$ & $\boldsymbol{E}_{\mathbf{e x p}} / \mathbf{c m}^{\mathbf{- 1}}$ & $\boldsymbol{f}$ \\
\hline $\mathbf{S}_{\mathbf{1}}$ & $\begin{array}{c}27370 \\
(29971)\end{array}$ & 27868 & $\begin{array}{c}0.040 \\
(0.04)\end{array}$ & $\begin{array}{c}27810 \\
(29955)\end{array}$ & 28043 & $\begin{array}{c}0.07 \\
(0.070)\end{array}$ \\
\hline $\mathbf{S}_{\mathbf{2}}$ & $\begin{array}{c}32730 \\
(35737)\end{array}$ & 32500 & $\begin{array}{c}0.074 \\
(0.097)\end{array}$ & $\begin{array}{c}34770 \\
(37052)\end{array}$ & $35100^{*}$ & $\begin{array}{c}0.0007 \\
(0.001)\end{array}$ \\
\hline $\mathbf{S}_{3}$ & $\begin{array}{c}42730 \\
(46247)\end{array}$ & 42230 & $\begin{array}{c}0.63 \\
(0.64)\end{array}$ & $\begin{array}{c}43420 \\
(46494)\end{array}$ & 41988 & $\begin{array}{c}0.65 \\
(0.71)\end{array}$ \\
\hline
\end{tabular}

Table 1. ADC(2) adiabatic excitation energies $\left(E_{0}\right)$ for protonated quinoline and isoquinoline (DFT calculations performed in reference 24 are enclosed in brackets) and oscillator strengths $(f)$. Experimental transition energies $\left(E_{\text {exp }}\right)$ are taken from band origins; $(*)$ very weak transition, the assignment of the band onset at $\sim 35100 \mathrm{~cm}^{-1}$ is tentative. Ground state geometry optimized in $\mathrm{C}_{1}$ point-group symmetry (MP2/cc-pVDZ).

The energetics of the low lying excited states of $\mathrm{QH}^{+}$and $\mathrm{iQH}^{+}$is very similar, in agreement with the good correspondence between their electronic spectra in Figures 2 and 4. In both molecular ions the excitation to the first excited state $\left(\mathrm{S}_{1}\right)$ involves a $\pi$-HOMO to an antibonding $\pi^{*}$-LUMO transition with a moderate oscillator strength, which can be perfectly assigned to the first band of the electronic spectrum (band onsets at $27868 \mathrm{~cm}^{-1}$ for $\mathrm{QH}^{+}$and $28043 \mathrm{~cm}^{-1}$ for $\left.\mathrm{iQH}^{+}\right)$. In the case of $\mathrm{QH}^{+}$, the next excitation $\left(\mathrm{S}_{2} \leftarrow \mathrm{S}_{0}\right)$ can largely be attributed to a (LUMO $\leftarrow$ HOMO-1) transition, for which the magnitude of the oscillator 
strength is significant. However, the $\mathrm{iQH}^{+} \mathrm{S}_{2} \leftarrow \mathrm{S}_{0}$ transition involves additional molecular orbitals (HOMO and LUMO+1) and the calculations predict almost no absorption for this transition. This is in agreement with the experiments $(\mathrm{m} / \mathrm{q}=77$ and 102 channels), in which a well-defined band starts at $\sim 32500 \mathrm{~cm}^{-1}$ for $\mathrm{QH}^{+}$, very close to the $E_{0}$ value calculated for $\mathrm{S}_{2}$, while only a very weak absorption at $\sim 35100 \mathrm{~cm}^{-1}$ appears in the case of iQH. Supplementary $\mathrm{S}_{1}$ and $\mathrm{S}_{2}$ optimizations have been performed starting from non-planar structures, which converged back to planar geometry. The third excitation $\left(\mathrm{S}_{3} \leftarrow \mathrm{S}_{0}\right)$ computed for $\mathrm{QH}^{+}$and $\mathrm{iQH}^{+}$lies in the deep UV region, showing $E_{0}$ values close to the observed band onsets at $42230 \mathrm{~cm}^{-1}$ and $41988 \mathrm{~cm}^{-1}$, respectively. Even though several molecular orbitals contribute to this transition in $\mathrm{iQH}^{+}$, the $(\mathrm{LUMO}+1 \leftarrow \mathrm{HOMO})$ transition prevails. According to our calculations, $\pi \sigma^{*}$ states are not energetically accessible. Under Cs-symmetry restriction, the optimized $\mathrm{S}_{1}$ excited state of $\mathrm{QH}^{+}$and $\mathrm{iQH}^{+}$are found of $\mathrm{A}^{\prime}\left(\pi \pi^{*}\right)$ type and the transition energies are the same as with the $\mathrm{C}_{1}$ symmetry calculation.

$\mathrm{S}_{1}$ and $\mathrm{S}_{3}$ low lying vibrational modes $\left(\tilde{v}_{\text {calc }}\right)$ calculated for $\mathrm{QH}^{+}$and $\mathrm{iQH}^{+}$are shown in Tables 2 and 3, respectively. The assignment of the experimental bands ( $\left.\tilde{v}_{\exp }\right)$ is done under the assumption that at the low temperature of the experiment the vibrational modes of the electronic ground state are not excited. We have restricted the list to frequencies lower than $1000 \mathrm{~cm}^{-1}$ and excluded a few unassigned experimental bands of weak intensity. Although most of calculated frequencies agree well with the measured values, in a few cases the assignment must be considered as tentative because $\left|\tilde{v}_{\exp }-\tilde{v}_{\text {calc }}\right|$ exceeds the experimental resolution of the apparatus (indicated with asterisks in the tables. The calculated in-plane vibrational modes $\tilde{v}_{28-33}$ are active in the $\mathrm{S}_{1}$ excited state of $\mathrm{QH}^{+}$under our experimental conditions, three of them being clearly assigned to intense spectral bands: $\tilde{v}_{\exp }=576 \mathrm{~cm}^{-1}$ $\left(\tilde{v}_{30}\right.$, nonsymmetrical ring deformation), $\tilde{v}_{\exp }=492 \mathrm{~cm}^{-1}$ ( $\tilde{v}_{31}$, symmetrical breathing), and $\tilde{v}_{\text {exp }}=481 \mathrm{~cm}^{-1}\left(\tilde{v}_{32}\right.$, nonsymmetrical ring deformation). The modes $\tilde{v}_{30}$ and $\tilde{v}_{31}$ are also active in the $\mathrm{S}_{3}$ excited state at slightly different frequencies (578 and $480 \mathrm{~cm}^{-1}$, respectively). The low energy out-of-plane mode $\tilde{v}_{47}$ is apparently active in the $\mathrm{S}_{3}$ state of $\mathrm{QH}^{+}$(two quanta match the band at $\tilde{v}_{\exp }=220 \mathrm{~cm}^{-1}$ ). The in-plane low frequency vibrations calculated for $\mathrm{iQH}^{+}$ do not differ much in energy from those of its structural isomer. In-plane $\mathrm{iQH}^{+}$vibrational modes $\tilde{v}_{28-33}$ are again active in $\mathrm{S}_{1}$, the same three modes, $\tilde{v}_{\text {exp }}=588 \mathrm{~cm}^{-1}\left(\tilde{v}_{30}\right.$, symmetrical ring deformation), $\tilde{v}_{\exp }=486 \mathrm{~cm}^{-1}\left(\tilde{v}_{31}\right.$, symmetrical breathing) and $\tilde{v}_{\exp }=467 \mathrm{~cm}^{-1}\left(\tilde{v}_{32}\right.$, 
nonsymmetrical breathing) prevailing. The modes $\tilde{v}_{30-32}$ appear to be active in the $\mathrm{S}_{3}$ excited state of $\mathrm{iQH}^{+}\left(\tilde{v}_{30}\right.$ at $656 \mathrm{~cm}^{-1}$ and a band comprising $\tilde{v}_{32}$ and $\tilde{v}_{31}$ at $456 \mathrm{~cm}^{-1}$ ), although the assignment is not conclusive due to the large experimental bandwidths. No out-of-plane modes are visible in the experimental spectra of $\mathrm{iQH}^{+}$.

\begin{tabular}{c|c|c}
\hline \multicolumn{3}{c}{$\mathbf{S}_{\mathbf{1}} \leftarrow \mathbf{S}_{\mathbf{0}}$} \\
\hline$\tilde{v}_{\exp }\left(\mathrm{cm}^{-1}\right)$ & $\tilde{v}_{\text {calc }}\left(\mathrm{cm}^{-1}\right)$ & assignment \\
\hline 354 & 353 & $33_{0}^{1}$ \\
\hline 481 & 488 & $32_{0}^{1}$ \\
\hline 492 & 493 & $31_{0}^{1}$ \\
\hline 576 & 579 & $30_{0}^{1}$ \\
\hline 755 & 763 & $29_{0}^{1}$ \\
\hline 784 & 793 & $28_{0}^{1}$ \\
\hline 971 & 976 & $32_{0}^{2}$ \\
\hline 988 & 986 & $31_{0}^{2}$ \\
\hline
\end{tabular}

\begin{tabular}{c|c|c}
\hline \multicolumn{3}{c}{$\mathbf{S}_{3} \leftarrow \mathbf{S}_{\mathbf{0}}$} \\
\hline$\tilde{v}_{\exp }\left(\mathrm{cm}^{-1}\right)$ & $\tilde{v}_{\text {calc }}\left(\mathrm{cm}^{-1}\right)$ & Assignment \\
\hline $230 / 244$ & 220 & $47_{0}^{2}\left(^{*}\right)$ \\
\hline 480 & 490 & $31_{0}^{1}$ \\
\hline 578 & 590 & $30_{0}^{1}$ \\
\hline 719 & 728 & $29_{0}^{1}$ \\
\hline 800 & 781 & $33_{0}^{1} 31_{0}^{1}+47_{0}^{2} 30_{0}^{1}\left(^{*}\right)$ \\
\hline 904 & 927 & $33_{0}^{1} 30_{0}^{1}(*)$ \\
\hline 941 & 960 & $32_{0}^{1} 31_{0}^{1}+47_{0}^{2} 29_{0}^{1}\left(^{*}\right)$ \\
\hline 980 & 980 & $31_{0}^{2}$ \\
\hline
\end{tabular}

Table 2. Experimental vibrational frequencies and calculated normal modes of $\mathrm{QH}^{+}$for the $\mathrm{S}_{1} \leftarrow \mathrm{S}_{\mathrm{o}}$ and $\mathrm{S}_{3} \leftarrow \mathrm{S}_{\mathrm{o}}$ transitions. (*) Tentative assignment.

\begin{tabular}{c|c|c}
\hline \multicolumn{3}{c}{$\mathbf{S}_{\mathbf{1}} \leftarrow \mathbf{S}_{\mathbf{0}}$} \\
\hline$\tilde{v}_{\text {exp }}\left(\mathrm{cm}^{-1}\right)$ & $\tilde{v}_{\text {calc }}\left(\mathrm{cm}^{-1}\right)$ & assignment \\
\hline 344 & 344 & $33_{0}^{1}$ \\
\hline 467 & 484 & $32_{0}^{1}\left(^{*}\right)$ \\
\hline 486 & 492 & $31_{0}^{1}$ \\
\hline 588 & 589 & $30_{0}^{1}$ \\
\hline 749 & 763 & $29_{0}^{1}$ \\
\hline 783 & 785 & $28_{0}^{1}$ \\
\hline 831 & 830 & $33_{0}^{1} 31_{0}^{1}$ \\
\hline 950 & 976 & $32_{0}^{1} 31_{0}^{1}\left(^{*}\right)$ \\
\hline 970 & 984 & $31_{0}^{2}$ \\
\hline
\end{tabular}

\begin{tabular}{c|c|c}
\hline \multicolumn{3}{c}{$\mathbf{S}_{\mathbf{3}} \leftarrow \mathbf{S}_{\mathbf{0}}$} \\
\hline$\tilde{v}_{\exp }\left(\mathrm{cm}^{-1}\right)$ & $\tilde{v}_{\text {calc }}\left(\mathrm{cm}^{-1}\right)$ & assignment \\
\hline 456 & 461 and 497 & $32_{0}^{1}$ and $31_{0}^{1}$ \\
\hline 656 & 599 & $30_{0}^{1}\left(^{*}\right)$ \\
\hline 903 & 922 & $32_{0}^{2}\left({ }^{*}\right)$ \\
\hline
\end{tabular}

Table 3. Experimental vibrational frequencies and calculated normal modes of $\mathrm{iQH}^{+}$for the $\mathrm{S}_{1} \leftarrow \mathrm{S}_{\mathrm{o}}$ and $\mathrm{S}_{3} \leftarrow \mathrm{S}_{\mathrm{o}}$ transitions. (*) Tentative assignment. 


\section{Discussion}

The fragmentation spectra of the molecular ions $\mathrm{QH}^{+}$and $\mathrm{iQH}^{+}$look very similar (compare Figures 2 and 4), exhibiting two intense band systems associated with the $\mathrm{S}_{1} \leftarrow \mathrm{S}_{0}$ and $\mathrm{S}_{3} \leftarrow \mathrm{S}_{0}$ absorptions (UV and deep-UV bands, respectively) in addition to a weak broad structure located in between, which has been assigned to the $\mathrm{S}_{2} \leftarrow \mathrm{S}_{0}$ absorption. The UV and deep-UV band origins correspond reasonably well with previous estimations done by action spectrometry at room temperature, ${ }^{24}$ although the low temperature of the ions achieved in our experiments allow us to observe well-defined $0-0$ bands and clear progressions of vibrational bands (Figures 3 and 5). In the UV and deep-UV range, the electronic spectra of $\mathrm{QH}^{+}$and $\mathrm{iQH}^{+}$are governed by the excitation of $\pi$ electrons. All the observed transitions are of $\pi \pi^{*}$ character because protonation transforms the $\mathrm{n}$ orbital (lone pair) on the nitrogen atom to a $\sigma$ orbital and the resulting $\sigma \pi^{*}$ transitions become too high to be observed. $\pi \sigma^{*}$ transitions are also calculated beyond experimental access due to their high energies.

In coincidence with previous DFT calculations, ${ }^{24}$ the RI-ADC(2) method also shows that the $\mathrm{S}_{1} \leftarrow \mathrm{S}_{0}$ excitation in both $\mathrm{QH}^{+}$and $\mathrm{iQH}^{+}$can be viewed to a great extent as a transition from the HOMO to the LUMO, while $\mathrm{S}_{2} \leftarrow \mathrm{S}_{0}$ and $\mathrm{S}_{3} \leftarrow \mathrm{S}_{0}$ excitations involve other orbitals. The probability density of the HOMO is somewhat larger on the non-protonated ring than on the heterocycle, whereas a more uniform distribution characterizes the LUMO. Consequently, a small degree of charge transfer (CT) is involved in the photoabsorption of quinolinium ions, in contrast to the structurally-akin protonated naphthalene $\left(\mathrm{NphH}^{+}\right)$for which the $\mathrm{S}_{1} \leftarrow \mathrm{S}_{0}$ excitation involves a clear transition of one electron from the non-protonated to the protonated aromatic ring. ${ }^{26}$ The $\mathrm{S}_{1} \leftarrow \mathrm{S}_{0}$ band origins of $\mathrm{QH}^{+}$and $\mathrm{iQH}^{+}$reported in Figures 2 and 4 are shifted about $0.5 \mathrm{eV}$ to lower energies with respect to the onsets of the $\pi \pi^{*}$ transition $\left(\mathrm{S}_{2} \leftarrow \mathrm{S}_{0}\right.$, in this case) of quinoline ${ }^{20}$ and isoquinoline. ${ }^{21}$ The observed spectral shifts to the red denote an enhancement of the proton affinity in the $S_{1}$ state, which is probably a common feature among polyaromatic hydrocarbons and $\mathrm{N}$-heterocycles.

An interesting observation comes out when the computed adiabatic excitation energies summarized in Table 1 are compared with the experimental results. Although the ADC(2) transition energies agree very closely with the observed band onsets, the computed oscillator strengths do not reproduce the relative band intensities found in the spectra. For example, one would expect larger $f\left(\mathrm{~S}_{1} \leftarrow \mathrm{S}_{0}\right)$ values in the calculations for both $\mathrm{QH}^{+}$and $\mathrm{iQH}^{+}$, considering 
that the experimental bands are very intense. This was also noted previously ${ }^{24}$ for the same species. An explanation for that might be that the oscillator strength effect on the band intensity can be to a great extent compensated by large Franck Condon factors.

The structural similarity of $\mathrm{QH}^{+}, \mathrm{iQH}^{+}$and $\mathrm{NphH}^{+}$can also be accounted for by plotting the vibrationally resolved photofragmentation spectra of these ions in the vicinity of the $\mathrm{S}_{1} \leftarrow \mathrm{S}_{0}$ band origin, as shown in Figure 6 . The low lying active vibrational modes $\tilde{v}_{28-33}$ of $\mathrm{QH}^{+}$and $\mathrm{iQH}^{+}$match reasonably well with the modes $\tilde{v}_{29-34}$ of $\mathrm{NphH}^{+}$(see supplementary information of reference 27). The first mode in the region of $\sim 340 \mathrm{~cm}^{-1}$ (indicated as $\tilde{v}_{33}$ for the protonated quinolines and $\tilde{v}_{34}$ for protonated naphthalene) correspond to the in-plane symmetric bending, while the mode at $485 \mathrm{~cm}^{-1}\left(\tilde{v}_{31}, \mathrm{QH}^{+}\right.$and $\left.\mathrm{iQH}^{+} ; \tilde{v}_{32} \mathrm{NphH}^{+}\right)$is a symmetric breathing, and the other modes involve different in-plane deformations of the carbon skeleton. The modes $\tilde{v}_{29-31}$ are also present in the $\mathrm{S}_{3} \leftarrow \mathrm{S}_{0}$ excitation.

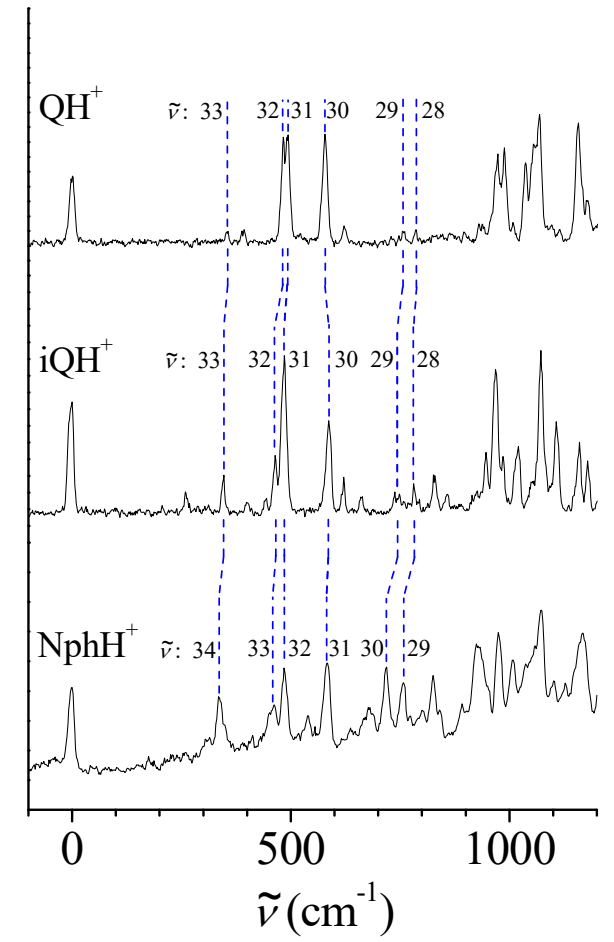

Figure 6. Comparison among the photofragmentation spectra of protonated quinoline $\left(\mathrm{QH}^{+}\right)$, isoquinoline $\left(\mathrm{iQH}^{+}\right)$and naphthalene $\left(\mathrm{NphH}^{+}\right)$in the vicinity of the $\mathrm{S}_{1} \leftarrow \mathrm{S}_{0}$ band origin. The numbers label the low lying active vibrational modes. 
The transition to the $\mathrm{S}_{2}$ state is observed broad and featureless in $\mathrm{QH}^{+}$and $\mathrm{iQH}^{+}$ spectra, probably because it is involved in a fast internal conversion process. However, according to our ab-initio calculations, the excited state $S_{2}$ does not lose its planar symmetry and this makes improbable that an out-of-plane non-radiative deactivation channel towards the ground state will take place. The reason for the absence of structure is not clear.

The $\mathrm{S}_{3} \leftarrow \mathrm{S}_{0}$ absorption in quinolinium ions is found practically selective to the fragmentation channel leading to the phenyl cation $(m / q=77)$ and it exhibits a fast deactivation rate estimated in terms of the experimental bandwidths (particularly for $\mathrm{iQH}^{+}$), which are much larger than the apparatus resolution. A question arises whether the photoexcitation to the $\mathrm{S}_{3}$ excited state induces the production of phenyl cations by secondary fragmentation of $m / q=103$ ions or alternatively a non-sequential fragmentation pathway takes place. Evidence supporting that the phenyl cation is not the daughter ion from the $m / q=$ 103 fragment arises by considering the different rate of formation of both ions. It was found that while the phenyl fragments are produced in a very short time (less than $100 \mathrm{~ns}$ ) after the fragmentation laser pulse, it takes several milliseconds for $m / q=103$ fragments to appear in the trap.

The ultrashort lifetime of the $\mathrm{S}_{3}$ excited state suggested by the bandwidths appearing in the deep-UV absorption of $\mathrm{iQH}^{+}$and, to a lower extent, $\mathrm{QH}^{+}$might originate from a nonradiative deactivation via isomerization processes like those occurring in other azabenzenes. For example, valence isomers such as Dewar and prefulvene structures have been proposed as intermediates associated to the 2-3 ps relaxation of the lowest $\pi \pi^{*}$ excited state measured for isolated pyridine ${ }^{36}$ and for protonated pyridine in solution ${ }^{37}$ by femtosecond time-resolved mass spectrometry and transient absorption, respectively. A conical intersection located by ab-initio calculations of the potential surfaces was found to play a determinant role in the isomerization pathway leading to the ultrafast deactivation. ${ }^{38}$ Dewar pyridines have also been produced by argon matrix isolation and UV irradiation and characterized by FT IR. ${ }^{39}$ Although the Dewar forms of protonated quinoline and isoquinoline are energetically accessible from their $\mathrm{S}_{3}$ excited state according to DFT/B3LYP calculations, no direct evidence on the formation of these photoisomers is derived from our experiments.

Finally, photoproducts and branching ratios do not change from $\mathrm{QH}^{+}$to $\mathrm{iQH}^{+}$and are consistent with those obtained by action spectroscopy at room temperature, which were extensively analyzed in reference 24 . 


\section{Conclusions}

The key result of the present work is the presentation of cold photofragmentation spectra of single-isomeric protonated quinoline and isoquinoline ions, together with ab-initio adiabatic transitions energies that nicely match with the experimentally well-defined band onsets. Although the second band in the electronic spectra does not show any vibrational structure, the first and the third absorptions show several in-plane vibrations that have been analyzed and assigned with the help of calculations performed in the ground and the excited states.

The bandwidths measured in the deep-UV absorption of $\mathrm{iQH}^{+}$and, to a lower extent, in $\mathrm{QH}^{+}$denote that the lifetime of the $\mathrm{S}_{3}$ excited state is in the subpicosecond time scale, possibly via a relaxation pathway involving valence photoisomers.

Finally, we have observed that the vibrational progressions in the $S_{1}$ excited state of $\mathrm{QH}^{+}$and $\mathrm{iQH}^{+}$look very similar and resemble that of the structurally related protonated naphthalene.

\section{Bibliography:}

1. Kozin, I. S.; Larsen, O. F. A.; de Voogt, P.; Gooijer, C.; Velthorst, N. H. Isomer-Specific Detection of Azaarenes in Environmental Samples by Shpol'skii Luminescence Spectroscopy. Anal. Chim. Acta 1997, 354, 181-187.

2. Blumer, M.; Dorsey, T.; Sass, J. Azaarenes in Recent Marine Sediments. Science 1977, $195,283-285$.

3. Pereira, W. E.; Rostad, C. E.; Garbarino, J. R.; Hult, M. F. Groundwater Contamination by Organic Bases Derived from Coal-Tar Wastes. Environ. Toxicol. Chem. 1983, 2, 283-294.

4. Bleeker, E. A.; Wiegman, S.; deVoogt, P.; Kraak, M.; Leslie, H. A.; de Haas, E.; Admiraal, W. Toxicity of Azaarenes. Rev. Environ. Contam. Toxicol.2002, 173, 39-83. 
5. Tucker, S. A.; Acree, W. E., Jr.; Upton, C. Polycyclic Aromatic Nitrogen Heterocycles. Part V: Fluorescence Emission Behavior of Select Tetraaza- and Diazaarenes in Nonelectrolyte Solvents. Appl. Spectrosc. 1993, 47, 201-206.

6. Ricca, A.; Bauschlicher, C. W.; Rosi, M. Mechanism for the Incorporation of a Nitrogen Atom into Polycyclic Aromatic Hydrocarbon Cations. Chem. Phys. Lett. 2001, 347, $473-480$.

7. Bernstein, M. P.; Mattioda, A. L.; Sandford, S. A.; Hudgins, D. M. Laboratory Infrared Spectra of Polycyclic Aromatic Nitrogen Heterocycles: Quinoline and Phenanthridine in Solid Argon and $\mathrm{H}_{2} \mathrm{O}$. Astrophys. J. 2005, 626, 909-918.

8. Hudgins, D. M.; Bauschlicher, C. W., Jr.; Allamandola, L. J. Variations in the Peak Position of the $6.2 \mu \mathrm{m}$ Interstellar Emission Feature: A Tracer of $\mathrm{N}$ in the Interstellar Polycyclic Aromatic Hydrocarbon Population. Astrophys. J. 2005, 632, 316-322.

9. Van der Zwet, G. P.; Allamandola, L. J. Polycyclic Aromatic Hydrocarbons and the Diffuse Interstellar Bands. Astron. Astrophys. 1985, 146, 76-80.

10. Léger, A.; d'Hendecourt, L. Are Polycyclic Aromatic Hydrocarbons the Carriers of the Diffuse Interstellar Bands in the Visible? Astron. Astrophys. 1985, 146, 81-85.

11. Crawford, M. K.; Tielens, A. G. G. M.; Allamandola, L. J. Ionized Polycyclic Aromatic Hydrocarbons and the Diffuse Interstellar Bands. Astrophys. J. 1985, 293, L45-L48.

12. Allamandola, L. J.; Tielens, A. G. G. M.; Barker, J. R. Interstellar Polycyclic Aromatic Hydrocarbons - The Infrared Emission Bands, the Excitation/Emission Mechanism, and the Astrophysical Implications. Astrophys. J. Suppl. Ser. 1989, 71, 733-775.

13. Tielens, A. G. G. M. Interstellar Polycyclic Aromatic Hydrocarbon Molecules. Annu. Rev. Astron. Astrophys. 2008, 46, 289-337. 
14. Mattioda, A. L.; Rutter, L.; Parkhill, J.; Head-Gordon, M.; Lee, T. J.; Allamandola, L. J. Near-Infrared Spectroscopy of Nitrogenated Polycyclic Aromatic Hydrocarbon Cations from 0.7 to $2.5 \mu \mathrm{m}$. Astrophys. J. 2008, 680, 1243-1255.

15. Alvaro Galué, H.; Pirali, O.; Oomens, J. Gas-phase Infrared Spectra of Cationized Nitrogen-Substituted Polycyclic Aromatic Hydrocarbons. Astron. Astrophys. 2010, 517, A15.

16. Boersma, C.; Bregman, J. D.; Allamandola, L. J. Properties of Polycyclic Aromatic Hydrocarbons in the Northwest Photon Dominated Region of NGC 7023. I. PAH Size, Charge, Composition, and Structure Distribution. Astrophys. J. 2013, 769, 117.

17. Dryza, V., Sanelli, J. A., Robertson, E. G., \& Bieske, E. J. Electronic Spectra of GasPhase Polycyclic Aromatic Nitrogen Heterocycle Cations: Isoquinoline ${ }^{+}$and Quinoline $^{+}$. J. Phys. Chem. A 2012, 116, 4323-4329.

18. Noble, J. A.; Dedonder, C.; Jouvet, C. The Electronic Spectra of Protonated PANH Molecules. Astron. Astrophys. 2015, 577, A79.

19. Wanna, J.; Bernstein, E. R. van der Waals Clusters of Pyridazine and Isoquinoline: The Effect of Solvation on Chromophore Electronic Structure. J. Chem. Phys.1987, 86, 6707-6716.

20. Hiraya, A.; Achiba, Y.; Kimura, K.; Lim, E. C. Identification of the Lowest Energy $n \pi *$ States in Gas-Phase Polycyclic Monoazines: Quinoline and Isoquinoline. J. Chem. Phys. 1984, 81, 3345-3347.

21. Hiraya, A.; Achiba, Y.; Kimura, K.; Lim, E. C. Multiphoton Ionization Photoelectron Spectroscopy of Molecular Excited States in Supersonic Jet: Low-Lying Electronic States of Isoquinoline. Chem. Phys. Lett. 1991, 185, 303-309. 
22. Bouwman, J.; Sztáray, B.; Oomens, J.; Hemberger, P.; Bodi, A. Dissociative Photoionization of Quinoline and Isoquinoline. J. Phys. Chem. A 2015, 119, 1127-1136.

23. Klærke, B.; Holm, A. I. S.; Andersen, L. H. UV Action Spectroscopy of Protonated PAH Derivatives. Methyl Substituted Quinolines. Astron. Astrophys. 2011, 532, A132.

24. Hansen, C. S.; Blanksby, S. J.; Trevitt, A. J. Ultraviolet Photodissociation Action Spectroscopy of Gas-Phase Protonated Quinoline and Isoquinoline Cations. Phys. Chem. Chem. Phys. 2015, 17, 25882.

25. Féraud, G.; Dedonder-Lardeux, C.; Jouvet, C.; Inokuchi, Y.; Haino, T.; Sekiya, R.; Ebata, T. Development of Ultraviolet-Ultraviolet Hole-Burning Spectroscopy for Cold Gas-Phase Ions. J. Phys. Chem. Lett. 2014, 5, 1236-1240.

26. Alata, I.; Omidyan, R.; Broquier, M.; Dedonder, C.; Dopfer, O.; Jouvet, C. Effect of Protonation on the Electronic Structure of Aromatic Molecules: NaphthaleneH ${ }^{+}$. Phys. Chem. Chem. Phys. 2010, 12, 14456-14458.

27. Alata, I.; Dedonder, C.; Broquier, M.; Marceca, E.; Jouvet, C.Role of the ChargeTransfer State in the Electronic Absorption of Protonated Hydrocarbon Molecules. J. Am. Chem. Soc.2010, 132, 17483-17489.

28. Alata, I.; Bert, J.; Broquier, M.; Dedonder, C.; Feraud, G.; Grégoire, G.; Soorkia, S.; Marceca, E.; Jouvet, C. Electronic Spectra of the Protonated Indole Chromophore in the Gas Phase. J. Phys. Chem. A 2013, 117, 4420-4427.

29. Kang, H.; Féraud, G.; Dedonder-Lardeux, C.; Jouvet, C. New Method for DoubleResonance Spectroscopy in a Cold Quadrupole Ion Trap and Its Application to UV-UV Hole-Burning Spectroscopy of Protonated Adenine Dimer. J. Phys. Chem. Lett. 2014, 5, $2760-2764$. 
30. Ahlrichs, R.; Bär, M.; Häser, M.; Horn, H.; Kölmel, C. Electronic Structure Calculations on Workstation Computers: The Program System Turbomole. Chem. Phys. Lett.1989, $162,165-169$.

31. Trofimov, B.; Stelter, G.; Schirmer, J. Electron Excitation Energies Using a Consistent Third-Order Propagator Approach: Comparison with Full Configuration interaction and Coupled Cluster Results. J. Chem. Phys. 2002,117, 6402-6410.

32. Taccone, M. I.; Féraud, G.; Berdakin, M.; Dedonder-Lardeux, C.; Jouvet, C.; Pino, G. A. Communication: UV Photoionization of Cytosine Catalyzed by $\mathrm{Ag}^{+} . J$ Chem Phys. 2015, $143,041103$.

33. Féraud, G.; Berdakin, M.; Dedonder, C.;Jouvet, C.; Pino, G. A. Excited States of ProtonBound DNA/RNA Base Homodimers: Pyrimidines. J. Phys. Chem. B 2015, 119, 22192228.

34. Féraud, G.; Esteves-López, N.; Dedonder-Lardeux, C.; Jouvet, C. UV Spectroscopy of Cold Ions as a Probe of the Protonation Site. Phys. Chem. Chem. Phys. 2015, 17, $25755-25760$.

35. Horai, H.; Arita, M.; Kanaya, S.; Nihei, Y.; Ikeda, T.; Suwa. K.; Ojima, Y.; Tanaka, K.; Tanaka, S.; Aoshima, K.; Oda, Y.; Kakazu, Y.; Kusano, M.; Tohge, T.; Matsuda, F.; Sawada, Y.; Yokota Hirai, M.; Nakanishi, H.; Ikeda, K.; Akimoto, N.; Maoka, T.; Takahashi, H.; Ara, T.; Sakurai, N.; Suzuki, H.; Shibata, D.; Neumann, S.; Iida, T.; Tanaka, K.; Funatsu, K.; Matsuura, F.; Soga, T.; Taguchi, R.; Saito, K.; Nishioka, T. J. MassBank: A Public Repository for Sharing Mass Spectral Data for Life Sciences. Mass Spectrom. 2010, 45, 703-714.

36. Zhong, D.; Diau, E. W.-G.; Bernhardt, T. M.; De Feyter, S.; Roberts, J. D.; Zewail, A. H. Femtosecond dynamics of valence-bond isomers of azines: transition states and conical intersections. Chem. Phys. Lett. 1998, 298, 129-140. 
37. Chachisvilis, M.; Zewail, A. H. Femtosecond Dynamics of Pyridine in the Condensed Phase: Valence Isomerization by Conical Intersections. J. Phys. Chem. A 1999, 103, 74087418 .

38. Srinivasan, R.; Feenstra, J. S.; Park, S.; Xu, T. S.; Zewail, A. H. Dark Structures in Molecular Radiationless Transitions Determined by Ultrafast Diffraction. Science 2005, $307,558-563$.

39. Kudoh, S.; Takayanagi, M.; Nakata, Munetaka. Dewar Pyridine Studied by Matrix Isolation Infrared Spectroscopy and DFT Calculations. J. Photochem. Photobiol. 1999, $123,25-30$. 